\title{
Photodynamic cancer therapy: the underlying laser characteristics
}

\begin{abstract}
Photodynamic therapy is a developing method for the treatment of a certain types of cancer. It is a serious contender to other known therapies. Detection and treatment of cancer can be done with photosensitizing substances and a light source. The ensuing simulations uncover some suitable parameters of the laser and the photosensitizing substance that would cure esophagus cancer.
\end{abstract}

Keywords: Cancer, Esophagus, Photodynamic therapy, Photofrin, Laser, Light, Phosphorescence
Volume 5 Issue 5 - 2017

Helene Painchaud, Laurianne Plouffe, Abigaelle Bolduc, Abdessamad Benhsaien Department of Mathematics, CEGEP de l'Outaouais, Gatineau Quebec, Canada

Correspondence: Abdessamad Benhsaien, Department of Mathematics, CEGEP de I Outaouais, Gatineau Quebec, Canada Email Abdessamad.Benhsaien@cegepoutaouais.qc.ca

Received: May 14, 2017 | Published: June 08, 2017

\section{Introduction}

Cancer cells multiply due to changes in genetic codes. Unless eliminated by apoptosis, such cells would duplicate rapidly. Esophageal cancer, in particular, develops mainly in the glandular cells that form the mucus or in the squamous cells of the mucosa. The observed symptoms include weight loss, pain during swallowing, sore throat as well as chest and back pains. When the tumor reaches $3 \mathrm{~cm}$ in diameter, the chances of survival are slim.

A photosensitizer (PS) is a substance composed of molecules that can occupy a higher level of excitation pursuant to a photon capture. Photofrin is the readily available and selected PS throughout this work despite its few inherent drawbacks namely, a fairly long in situ reminiscent time of up to three weeks following the injection and a low selectivity for tumor cells. Blue light can detect cancer whereas red light can treat the same. The latter unique attribute, chiefly due to the absorption spectral profile in the first NIR optical window, means that red light - around $700 \mathrm{~nm}$ - can penetrate deep enough into the tissue while suffering negligible attenuation. The red light promotes the PS from its ground state to a higher state of excitation (singlets and triplets). The molecules in the triplet state return to the ground state via phosphorescence, a long radiative relaxation mechanism allowing to produce singlet oxygen. Finally, the reaction of the biomolecules and singlet oxygen is conducive to the destruction of the tumor cells .'

An ad-hoc light source that can cater for specific therapeutic traits is instrumental to the success of PDT. Two noteworthy requirements are a low-line width laser emission at the specified (blue/red) wavelength and a pre-engineered laser dynamics - such as a tailored emission lifetime.$^{2}$ - stand out. Moreover, in a non-topical light delivery, the coupling loss from the laser cavity onto the fiber pig-tails needs to be carefully considered.

\section{Photofrin Simulation}

SimphoSOFT software, a courtesy of Simphotec . ${ }^{3}$ successfully computes the Photofrin eigenenergies as depicted in Figure 1.

The injected red light promotes the PS from its lowest state S0 to the first excited state S1 (singlet stimulation S01), cross-transfers energy onto the triplet state T3 (W13), causing it to return to the highly reactive singlet ground state S0 (absorption A30). Such is the transition of interest (phosphorescence) with a lifetime of $368 \mathrm{~ns}$ in comparison to the absorption A10 with lifetime of $2.72 \mathrm{~ns}$.

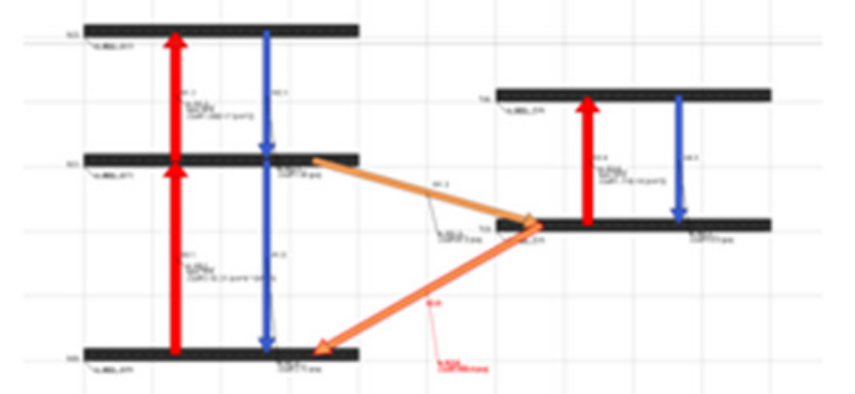

Figure I Photofrin (left) and $\mathrm{O}_{2}$ (right) Energy Level Diagram.

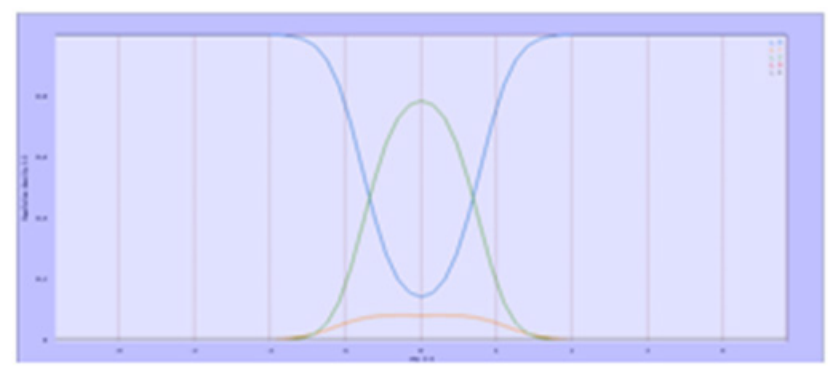

Figure 2 Distribution of State Level Probability of Occupancy.

Figure 2 depicts the spatial distribution, around the beam centre, of the probability of occupancy of the ground (blue), first excited singlet (green) and second excited singlet (orange) states, respectively. As expected, Figure 2 shows that near the beam centre where the light is mostly focused, the excited state is likely to host the photo-activated PS inducing a more probable phosphorescence.

\section{Laser Simulation}

Simphosoft succesfully simulates the required laser emission with the Photofrin at a selected wavelength of $630 \pm 3 \mathrm{~nm}$. Such a spectral uncertainty, $\pm 3 \mathrm{~nm}$, may trigger the onset of side longitudinal modes which are known to alter the laser performance. Albeit, Figure 3 depicts a single-mode emission with a quite reasonable SMSR (sidemode suppression ratio), unfortunately, not estimated by Simphosoft.

In order to alleviate such a nuisance, the laser source needs to embed a spectral filter (e.g. distributed feedback grating) into its 
cavity. Such a premise for tune-ability promises vigor and rigor in reducing the off-set between the material gain peak and the cavity carrier wavelength. The size of the laser sample has a significant impact on the graph shown in Figure 3. The simulations have shown that an optimal sample of $0.2 \mu \mathrm{m}$ by $0.2 \mu \mathrm{m}$ is good enough for the spectral components to resolve, thereby, yielding a beam waist (FWHM) of $21 \mathrm{~nm}(0.175 \mathrm{~ns})$.

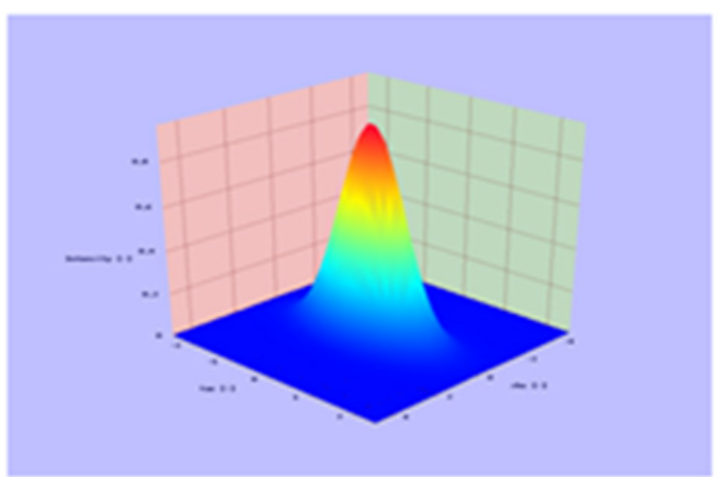

Figure 3 Light Intensity Spatial and Temporal Profile.

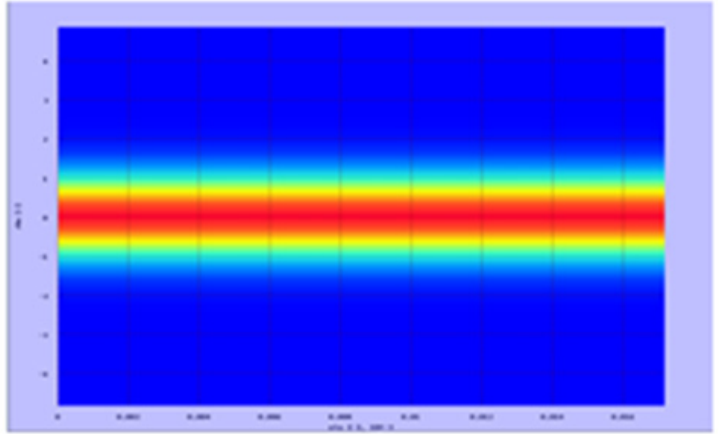

Figure 4 Light Beam Gaussian Divergnece.
Furthermore, a beam divergence in the order of few nanometers, as depicted in Figure 4, hinders the treatment effectiveness since the laser sample would be all too small compared to the size of the tumor. This entails that repeated applications are then required. The light intensity is shown, in Figure 4, to reach up to 0.016 pm in skin depth rendering the light therapy possible.

\section{Conclusion}

In theory, Photofrin proves to be effective against esophagus cancer. However, it remains yet to be tested in practice. Similar investigations need to consider other types of cancers and photosensitizer. It should be emphasized that the need for the development of an applicationspecific laser is critical to the success of PDT.

\section{Acknowledgements}

All co-authors wish to thank Simphotek for allowing to use their SimphoSOFT software.

\section{Conflicts of interest}

None.

\section{References}

1. Potasek M, Parilov E, Beeson $\mathrm{K}$ Calculation of singlet oxygen formation from one photon absorbing photosensitizers used in PDT. Optical Methods for Tumor Treatment and Detection: Mechanisms and Techniques in Photodynamic Therapy XXII, 85681D. 2013

2. Benhsaien A, Z Lu, K Hinzer, TJ Hall The Line width Broadening Factor: A Length Scale-Dependent Analytical Approach. American Journal of NanoResearch and Applications. 2017;5(1):1-6.

3. Simphotek, Inc. USA. 